\title{
Predicting Bank Interests When Monetary Rates Are Close to Zero
}

\author{
Laura Parisi'1, Igor Gianfrancesco ${ }^{2}$, Camillo Giliberto 3 , Paolo Giudici ${ }^{1}$ \\ ${ }^{1}$ Department of Economics and Management, University of Pavia, Pavia, Italy \\ ${ }^{2}$ Banco di Desio e della Brianza, Risk Management Division, Desio, Italy \\ ${ }^{3}$ Banca Monte dei Paschi di Siena, Siena, Italy \\ Email: laura.parisi01@universitadipavia.it, igianfrancesco@luiss.it, camillogiliberto@gmail.com, \\ paolo.giudici@unipv.it
}

Received 8 September 2015; accepted 8 January 2016; published 11 January 2016

Copyright @ 2016 by authors and Scientific Research Publishing Inc.

This work is licensed under the Creative Commons Attribution International License (CC BY).

http://creativecommons.org/licenses/by/4.0/

(c) (i) Open Access

\begin{abstract}
Monetary policies, either actual or perceived, cause changes in monetary interest rates. These changes impact the economy through financial institutions, which react to changes in the monetary rates with changes in their administered rates, on both deposits and lendings. The dynamics of administered bank interest rates in response to changes in money market rates is essential to examine the impact of monetary policies on the economy. Chong et al. (2006) proposed an error correction model to study such impact, using data previous to the recent financial crisis. In this paper we examine the validity of the model in the recent time period, characterized by very low monetary rates. The current state of close-to-zero monetary rates is of particular relevance, as it has never been studied before. Our main contribution is a novel, more parsimonious, model and a predictive performance assessment methodology, which allows comparing it with the error correction model.
\end{abstract}

\section{Keywords}

Error Correction Model, Forecasting Bank Rates, Monte Carlo Predictions, Interest Rate Risk Models

\section{Introduction}

Monetary policies, such as variations in the official rate or liquidity injections, cause changes in monetary interest rates. These changes impact the economy mainly in an indirect way, through financial institutions, which react to changes in the monetary rates with changes in their administered rates, on both deposits and lendings.

The dynamics of administered bank interest rates in response to changes in money market rates is essential to 
examine the impact of monetary policies on the economy. This dynamics has been the subject of an extensive literature; the available studies differ, depending on the used models, the period under analysis and the geographical reference.

The relationship between market rates and administered rates is a complicated one and the evidence presented, thus far, is mixed and inconclusive. [1], for example, examines the deposit rate setting behaviour of commercial banks in the United States and finds that a) banks in more concentrated markets exhibit greater rates rigidity; b) larger banks exhibit less rates rigidity; and c) deposit rates are more rigid upwards than downwards. [2], similarly, finds that deposit rates are more rigid when they are below their equilibrium level than when they are above; his finding on lending rate adjustment, however, is mixed. [3] examines how the lending and deposit rates of four banks and three building societies respond to changes in the base rate set by the Bank of England and finds that a) there is very little evidence on the asymmetric nature of adjustments in both the deposit and lending rates; b) there is no systematic difference in the administered rate pricing dynamics of banks and building societies; and c) the adjustment speed for deposit rates is, on average, roughly the same as that for loan rates.

More recent papers on the same issue include: [4]-[12]. Among them, [5] and [8], who apply and extend [13] error correction model, have become reference papers, in both the academic and the professional field.

The empirical evidence contained in all the previous papers can be summarized in the following points: a) bank rates react with a partial and delayed change to changes in the monetary rates; $b$ ) the speed and the degree to which they follow these changes present substantial differences between the various categories of banking products and between different countries.

The previous conclusions have been obtained for a relatively stable time period, previous to the emergence of the recent financial crisis.

After 2008, however, we have witnessed substantial changes. From a macroeconomic viewpoint, monetary interest rates are now, in most developed economies, close to zero, or negative; from a microeconomic viewpoint, bank management has changed substantially, for the compression of interest margins and for the increase in regulatory capital requirements. The effects of the previous changes on the transmission of monetary policies have not been yet fully investigated. In particular, the current state of close-to-zero interest rates is of particular relevance, as it has never been studied before.

When monetary rates are close to zero, the error correction model, albeit formally elegant, does not well capture the dynamic of administered rates, which appears strongly inertial.

The need of adapting the error correction model to the current situation is very relevant, not only from a macroeconomic point of view, but also from a microeconomic bank perspective and, in particular, in the measurement of interest rate risk, and in the related asset and liability management policies. We refer to [14] for further details.

The aim of this paper is to broaden the error correction model of [5], in a predictive performance comparison framework. Our results show that the error correction model performs quite well in a predictive sense. We also show that a more parsimonious model, described by only one equation, rather than two, is not inferior in terms of predictive performance, and, therefore, represents a valid alternative.

Our proposed methods are applied to data from the recent period (1999-2014), of a southern European country, with a traditional banking sector: Italy.

The paper is structured as follows. Section 2 describes the proposed models and, in particular: Section 2.1 describes the error correction model; Section 2.2 motivates and introduces the new proposed model; Section 2.3 provides the predictive performance environment used to compare the two models; Section 2.4 presents our proposal for the allocation of on demand deposits. Section 3 shows the empirical evidence obtained from the application of the models and, in particular: Section 3.1 describes the available data; Section 3.2 presents the estimation results obtained when the models are applied to such data; Section 3.3 compares the models in predictive performance. Finally, Section 4 concludes with some final remarks ${ }^{1}$.

\section{Methodology}

\subsection{The Error Correction Model}

In line with the contribution of [5], the relationship between monetary rates and administered bank rates can be

${ }^{1}$ A more extended version of this paper can be found at http://economia.unipv.it/docs/dipeco/quad/ps/RePEc/pav/demwpp/DEMWP0101.pdf. 
analyzed with the use of the Error Correction Model (ECM), following the procedure proposed by [13]. The model is based on two equations. A long-run relationship provides a measure of how a change in the monetary rate is reflected in the bank rate. A short-run equation, which includes an error correction term, analyzes variations of the administered interest rates as a function of variations in the monetary rates.

Indeed, [5] extended [13] by allowing the effect of the error correction term to depend on its sign. Their complete model can be formalized as follows:

$$
\left\{\begin{array}{l}
B R_{t}=k+\beta \cdot M R_{t}+\epsilon_{t} \\
\Delta B R_{t}=\alpha \cdot \Delta M R_{t}+\delta_{1}\left(B R_{t-1}-\beta \cdot M R_{t-1}-k\right)+\delta_{2}\left(B R_{t-1}-\beta \cdot M R_{t-1}-k\right)+u_{t},
\end{array}\right.
$$

where

$$
\begin{aligned}
& \begin{cases}\delta_{1}=0 & \text { if } B R_{t-1}-\beta \cdot M R_{t-1}-k<0, \\
\delta_{2} \neq 0 & \text { otherwise; }\end{cases} \\
& \begin{cases}\delta_{2}=0 & \text { if } B R_{t-1}-\beta \cdot M R_{t-1}-k>0, \\
\delta_{1} \neq 0 & \text { otherwise }\end{cases}
\end{aligned}
$$

In Equation (1) $B R_{t}$ and $M R_{t}$ represent, respectively, the bank administered rates and the monetary rates at time $t ; \quad \beta$ is a regression coefficient that gives a measure of the extent of the monetary rate transmitted on bank rates in a long-term perspective: in the case of $\beta=1$, the whole monetary rate is transmitted on the administered rate, while a value between 0 and 1 means that only a partial transmission mechanism occurs; $k$ is a constant that synthetizes all other factors that, in addition to the dynamics of monetary rates, may affect the transmission mechanism of the monetary policy on bank rates as, for example, the market power and the efficiency of a bank; $\epsilon$ is the error term of the long-run equation; $\delta_{1}$ and $\delta_{2}$ represent the adjustment speeds converge towards the equilibrium level; finally, $u_{t}$ is the error term of the short-run equation.

\subsection{The Proposed Model}

The aim of this subsection is to propose a bank rate model that, while based on the ECM, is more parsimonious and, therefore, easier to interpret and manage. To achieve this aim we examine the main components of the error correction model, so to establish a statistical methodology for their simplification.

First, it is of interest to check whether the assumption of a double error correction coefficient, introduced by [5], is justified and strictly necessary. To check this point the previous model can be compared, in a hypotheses testing framework, with the following nested model:

$$
\left\{\begin{array}{l}
B R_{t}=k+\beta \cdot M R_{t}+\epsilon_{t} \\
\Delta B R_{t}=\alpha \cdot \Delta M R_{t}+\delta\left(B R_{t-1}-\beta \cdot M R_{t-1}-k\right)+u_{t}
\end{array}\right.
$$

Differently from Equation (1), the model in (2) contains only one adjustment speed, so it does not admit the possibility of an asymmetric convergence of the administered interest rate to its equilibrium level.

Second, the error correction model contains one equation for the level of administered interest rates, and one for its variations. The two can be analyzed separately, with the simple regression models:

$$
\begin{aligned}
& B R_{t}=k+\beta \cdot M R_{t}+\epsilon_{t} \\
& \Delta B R_{t}=k+\beta \cdot \Delta M R_{t}+u_{t} .
\end{aligned}
$$

While model (3) explains the levels of banking rates in terms of the level of monetary ones, Equation (4) is a model for the variations of bank rates in terms of the variations of monetary rates. These models, albeit very simple, should be considered in practical applications, and compared in predictive performance with the error correction model, to check whether the latter can be simplified.

We anticipate that the above models are too simple to lead to a good predictive performance. However, the idea of replacing the error correction model with a one-equation one is tempting and, therefore, we now propose a one equation model that can be a valid competitor of the ECM. To achieve this aim we first examine the economic rationales behind the relationship we would like to investigate.

From a microeconomic viewpoint, as deposits are saving tools in competition with other instruments (such as 
bonds), it seems quite reasonable to assume that banks decide on the administered rate looking primarily at its level. Starting from the level, one can always obtain its variation through differentiation. A second consideration concerns the determinants of administered bank levels. Again, it is reasonable to think that bank deposit rates depend on both the level and on the variation of monetary rates. A third assumption, particularly important when monetary rates are close to zero, is that the level of deposit rates depends on the previous level of the same quantity, to allow for a slow and partial reaction to monetary rate changes, given that deposit rates affect considerably the income of a bank.

A macroeconomic perspective confirms the previous assumption: in particular, that is correct to consider, as a response variable, the level of the administered rate and not its variations. This because the relevant response variable for an expansion/restriction effect on the economy is represented by the level of the rates; on the explanatory side, we can model administered rate levels as a function of changes in the monetary rates, but also of their levels, which remain important even when close to zero.

On the basis of the above economic rationales, our proposed model is the following:

$$
B R_{t}=k+\beta \cdot M R_{t-1}+\gamma \cdot \Delta M R_{t}+\delta \cdot B R_{t-1}+\epsilon_{t} .
$$

The proposed model can be equivalently written in terms of the variations of the administered rates:

$$
\Delta B R_{t}=k+\beta \cdot M R_{t-1}+\gamma \cdot \Delta M R_{t}+(\delta-1) \cdot B R_{t-1}+\epsilon_{t}^{\prime} .
$$

To improve interpretability, the proposed model can also be expressed in a differential form:

$$
\frac{\mathrm{d} B R}{\mathrm{~d} s}=\beta \cdot\left[\frac{\mathrm{d} M R}{\mathrm{~d} s}\right]_{s=t}+\gamma \cdot\left[\frac{\mathrm{d}^{2} M R}{\mathrm{ds}^{2}}\right]_{s=t}+\gamma \cdot\left[\frac{\mathrm{d} B R}{\mathrm{ds}}\right]_{s=t-1} .
$$

The previous equation shows that the model can be interpreted as a "physical" description of the banking behavior in terms of deposit interest rates through its differentiation: the derivative of the bank administered rate depends both on the speed and on the acceleration/deceleration of monetary rates, as well as on the derivative of the administered rate with respect to its level in the previous time.

Note that the proposed model can be directly compared with the ECM with one adjustment speed. Comparing Equation (2) and Equation (5) it is clear that our proposal is a particular case of the latter, with some constraints on the parameters. By using the notational index 1 for the coefficients of the one-speed ECM and the index 2 for the coefficients referred to the proposed model, such constraints are the following:

$$
\left\{\begin{array}{l}
-\delta_{1} k_{1}=k_{2}, \\
-\delta_{1} \beta_{1}=\beta_{2}, \\
\alpha_{1}=\gamma_{2}, \\
\delta_{1}+1=\delta_{2} .
\end{array}\right.
$$

Note, in particular, that the last equation in (8) implies that $(\delta-1)$ represents the adjustment speed to which bank administered rates react to changes in the monetary rates, equivalently as the parameters $\delta_{1}$ and $\delta_{2}$ of the Error Correction Model in [5].

A full comparison of our model with the ECM cannot be easily carried out in a statistical testing framework, as the two models are, evidently, not nested; however, they can be compared in terms of predictive performance and, for this purpose, the next subsection introduces an appropriate methodology.

A different comparison between the two models can be carried out by looking at their time dynamics. This is of particular interest in the context of interest rate risk modeling. For sake of simplicity we illustrate this comparison for the first three one-month rates and, then, for the general situation.

For the error correction model, we consider the case of $\delta_{1} \neq 0$; the other case of $\delta_{2} \neq 0$ can be obtained analogously, replacing $\delta_{1}$ with $\delta_{2}$. Then, assume that:

$$
\left\{\begin{array}{l}
\delta_{1} \neq 0, \\
B R(0)=B R_{0}, \\
M R(0)=M R_{0}
\end{array}\right.
$$

then, for the first month ahead: 


$$
B R_{1}=B R_{0}+\Delta B R_{1}=B R_{0}\left(1+\delta_{1}\right)+\alpha \Delta M R_{1}-\delta_{1} \beta M R_{0}-\delta_{1} k
$$

For the second and the third month ahead, instead, we obtain:

$$
\begin{aligned}
B R_{2}= & B R_{1}+\Delta B R_{2}= \\
& B R_{0}\left(1+\delta_{1}\right)^{2}+\Delta M R_{1}\left(\alpha+\delta_{1} \alpha-\delta_{1} \beta\right) \\
& -\delta_{1} \beta M R_{0}\left(2+\delta_{1}\right)+\alpha \Delta M R_{2}-2 \delta_{1} k ; \\
B R_{3}= & B R_{0}\left(1+\delta_{1}\right)^{3}+\Delta M R_{1}\left[\alpha+\delta_{1}(\alpha-\beta)\left(2+\delta_{1}\right)\right] \\
& -M R_{0} \delta_{1} \beta\left[\left(1+\delta_{1}\right)\left(2+\delta_{1}\right)+1\right]+\Delta M R_{2}\left(\alpha-\delta_{1} \beta\right)-\delta_{1} k\left(3+2 \delta_{1}\right) .
\end{aligned}
$$

For our proposed model, assuming the same initial values $B R_{0}$ and $M R_{0}$ for the bank and the monetary interest rates, we find the following equation for the first month ahead:

$$
B R_{1}=M R_{0} \beta+\Delta M R_{1} \gamma+B R_{0} \delta+k
$$

whereas for the second and the third month ahead we obtain:

$$
\begin{aligned}
B R_{2}= & M R_{0} \beta(1+\delta)+\Delta M R_{1}[\beta+\delta \gamma]+\Delta M R_{2} \gamma+B R_{0} \delta^{2}+k(1+\delta) ; \\
B R_{3}= & M R_{0} \beta\left(1+\delta+\delta^{2}\right)+\Delta M R_{1}[\beta+\delta(\beta+\delta \gamma)] \\
& +\Delta M R_{2}[\beta+\delta \gamma]+\Delta M R_{3} \gamma+B R_{0} \delta^{3}+k \delta(1+\delta) .
\end{aligned}
$$

From the above calculations we can derive a general iterative formula for both models, in order to calculate bank interest rates at any time $t\left(B R_{t}\right)$, as functions of the levels of bank rates at time $t-1\left(B R_{t-1}\right)$.

For the error correction model such iterative equation is:

$$
B R_{t}=B R_{t-1}\left(1+\delta_{1}\right)-\delta_{1} \beta\left[M R_{0}+\sum_{s=1}^{t-1} \Delta M R_{s}\right]+\alpha \Delta M R_{t}-\delta_{1} k
$$

Similarly, for our proposed model we obtain:

$$
B R_{t}=\delta B R_{t-1}+\beta\left[M R_{0}+\sum_{s=1}^{t-1} \Delta M R_{s}\right]+\gamma \Delta M R_{t}+k
$$

\subsection{Predictive Performance Assessment}

While the assumption of a double error correction coefficient can be easily tested against a one error correction model, other simplifications of the ECM model require a more general set-up. This can be provided, for example, by a predictive performance framework that we are going to illustrate in this subsection. Doing so, we can enrich the error correction model with a validation procedure that is, to our knowledge, not yet available in the literature.

In order to predict bank rates, we need to estimate reasonable future values of the monetary rates. Consistently with the literature, we assume that their variation follows a Wiener process.

More formally, assume that we want to predict the level of monetary rates for each of the next 12 months. Let $\widehat{\triangle M R}_{i}$ indicate the variation of the monetary rate in a given month. We then assume that $\widehat{\triangle M R}_{i}$ are independently and identically distributed Gaussian random variables, so that:

$$
\left\{\begin{array}{l}
\widehat{\Delta M R} \sim N\left(0, \sigma^{2}\right) \\
\widehat{M R}_{i}=\widehat{M R}_{i-1}+\widehat{\Delta M R}_{i} \quad i=1, \cdots, 12 .
\end{array}\right.
$$

Equation (11) describes a recursive procedure to obtain predictions of the monetary rates for a given year ahead, based on the Wiener process assumption. We can then insert the predicted monetary rates as regressor values in the models of the previous subsection and, thus, obtain predictions for the administered bank rates. In particular, for model (1) we obtain:

$$
\left\{\begin{array}{l}
\widehat{B R}_{i}=\widehat{B R}_{i-1}+\widehat{\triangle B R}_{i}, \\
\widehat{\Delta B R}_{i}=\alpha \cdot \widehat{\triangle M R}_{i}+\delta_{1}\left(\widehat{B R}_{i-1}-\beta \cdot \widehat{M R}_{i-1}-k\right)+\delta_{2}\left(\widehat{B R}_{i-1}-\beta \cdot \widehat{M R}_{i-1}-k\right)
\end{array}\right.
$$


where

$$
\begin{aligned}
& \begin{cases}\delta_{1}=0 & \text { if } \widehat{B R}_{i-1}-\beta \cdot \widehat{M R}_{i-1}-k<0, \\
\delta_{2} \neq 0 & \text { otherwise; }\end{cases} \\
& \begin{cases}\delta_{2}=0 & \text { if } \widehat{B R}_{i-1}-\beta \cdot \widehat{M R}_{i-1}-k>0, \\
\delta_{1} \neq 0 & \text { otherwise. }\end{cases}
\end{aligned}
$$

For model (5) we obtain that:

$$
\widehat{B R}_{i}=k+\beta \cdot \widehat{M R}_{i-1}+\gamma \cdot \widehat{\triangle M R}_{i}+\delta \cdot \widehat{B R}_{i-1} .
$$

According to the standard cross-validation (backtesting) procedure, to evaluate the predictive performance of a model, we can compare, for a given time period, the predictions of monetary rates obtained with the previous equations with the actual values. To obtain a robust measurement we can indeed generate $N$ scenarios of monetary rates, using (11), and obtain the corresponding bank rates, using either (1) or (5). On the basis of them we can calculate and approximate Monte Carlo expected values and variances of the predictions, as follows.

Let $Y$ be a bank rate to be predicted at time $i$, with unknown density function $f_{Y}(y)$. The expected value of $Y$ can then be approximated with

$$
\widehat{\mathbb{E}(Y)}=\frac{1}{N} \sum_{k=1}^{N} y^{(k)}
$$

and its variance with

$$
\widehat{\operatorname{var}(Y)}=\frac{1}{N^{2}} \sum_{k=1}^{N}\left[y_{i}-\hat{E}(Y)\right]^{2} .
$$

In the next section we will use (12) and (13) to compare model predictive performances. Before proceeding, we would like to remark that the random number generation at the basis of the Monte Carlo algorithm is pseudorandom, and depends on an initial seed. Different seeds may lead to different results so that models can not be compared equally. We have thus decided to use the same random seed for all models, so that the differences in performances are not biased by the Monte Carlo random mechanism.

\section{Data Analysis and Results}

\subsection{Descriptive Analysis}

The recent financial crisis has had a major impact on the banking sector and, in particular, has led to a change in the relationship between monetary and administered rates and, therefore, to the transmission mechanisms of monetary policies. In the Eurozone, characterized by one monetary authority (the European Central Bank) that regulates still fragmented national markets (see [15]), this effect is particularly evident: southern European countries, differently from what expected, have benefited very little from the drop of monetary rates that has followed the financial crisis.

To investigate the above issues we focus on a southern European country, Italy, for which the transmission of monetary impulses is particularly problematic, given the importance of the banking sector and the difficult economic situation.

Accordingly, we have collected monthly time series data on monetary rates and on aggregate bank deposits administered rates from the statistical database provided by the Bank of Italy, for the period ranging from January 1999 to December 2014.

For the purposes of our analysis, the monetary rate used in this paper is the 1-month Euribor. This choice has been based on the fact that this rate has a greater correlation with the administered bank rate with respect to the other monetary rates, such as the EONIA and the Euribor at 3 and 6 months, as can be seen in Table 1 .

Figure 1 represents the time series of the chosen monetary rates, along with that of the aggregate administered bank rates on deposits, for the considered time period.

From Figure 1 note that both the administered and the monetary rates rapidly decreased in 2008 and 2009, while in the last two years they have remained quite stable and close to zero. Moreover, the two curves seem to have the same shape between 1999 and 2008, while the relationship between the two radically changes in the 
Table 1. Correlation matrix between the EONIA rate, the Euribor rates and the Bank administered rate.

\begin{tabular}{cccccc}
\hline & EONIA & Euribor $(1 \mathrm{~m})$ & Euribor $(3 \mathrm{~m})$ & Euribor $(6 \mathrm{~m})$ & Bank rate \\
\hline EONIA & 1.0000 & & & & \\
Euribor $(1 \mathrm{~m})$ & 0.9904 & 1.0000 & & & \\
Euribor $(3 \mathrm{~m})$ & 0.9801 & 0.9951 & 1.0000 & 0.9972 & 1.0000 \\
Euribor $(6 \mathrm{~m})$ & 0.9701 & 0.9876 & 0.9453 & 0.9333 & 1.0000 \\
Bank rate & 0.9488 & 0.9512 & & \\
\hline
\end{tabular}

Bank administered interest rates and Monetary rates

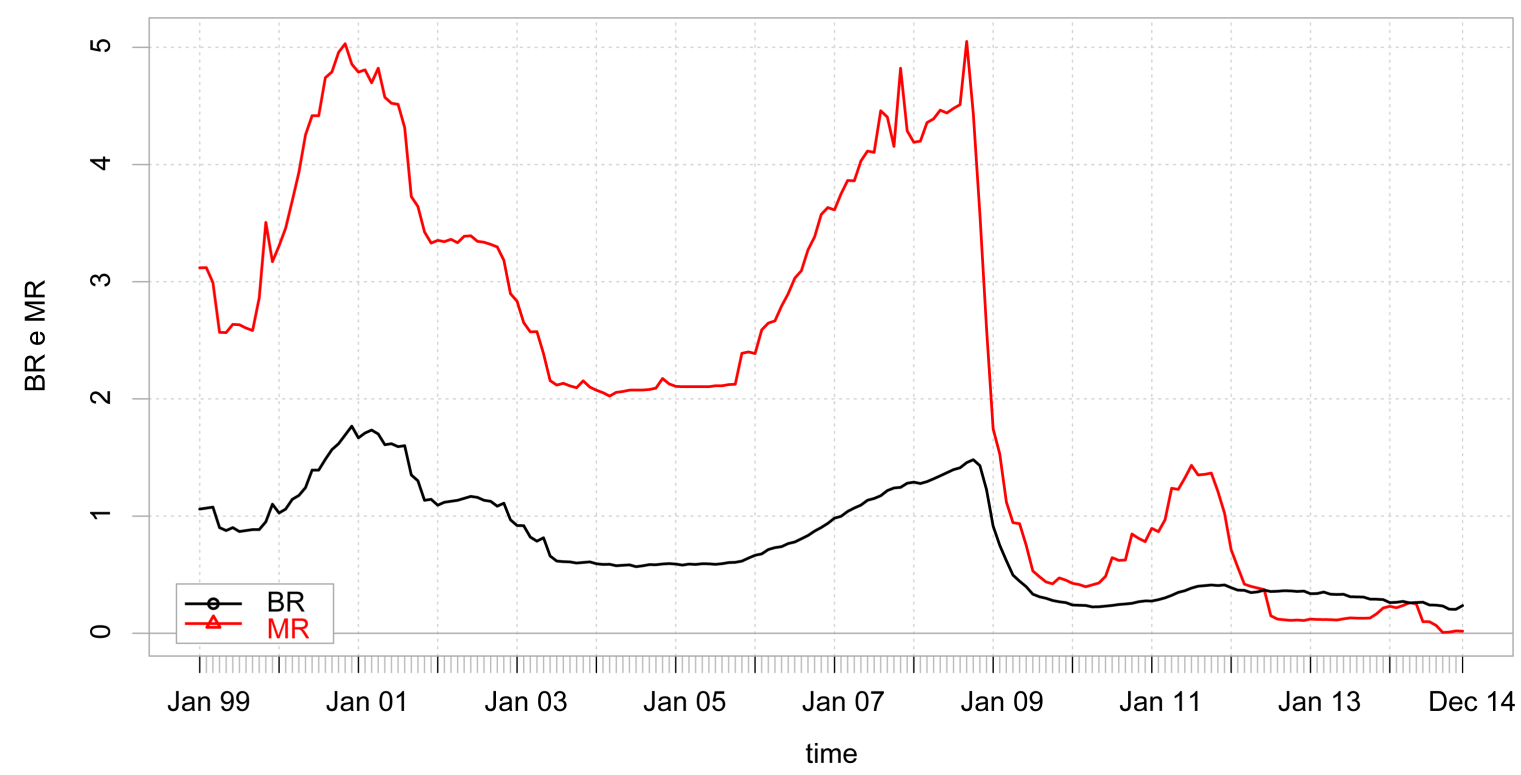

Figure 1. The observed monetary and administered bank rates.

following years, leading to overlaps and different behaviors. In other words, the correlation pattern between the bank administered rate and the monetary rate shows a very heterogeneous behavior: before 2008 they seem to have a stable relationship; in 2008 they both dropped; after that time they look stable and close to zero, with a relationship that is indeed quite different from the one observed before the crisis.

To obtain further insights, in Figure 2 we present the histogram and the corresponding density estimate of the two rates.

Figure 2 reveals that bank administered interest rates are more concentrated around their mean value, while monetary rates are quite spread.

It is also interesting to compare the distributions of the variations of the two rates, represented in Figure 3.

From Figure 3 note that the variations of the administered bank rates are more concentrated around zero, while monetary rates seem to have broader variations. Indeed, the behavior of $\triangle M R$ justifies the assumption of considering the variations of monetary interest rates as a Wiener process, so that they can be modeled according to Equation (11).

We have previously commented on the change in the relationship between the two rates, comparing the situation before and after 2009. This switching behavior can be easily seen by looking at the correlation between the rates and their variations. Table 2 shows the correlations between the rates and between their variations in the two periods (1999-2008) and (2009-2014), before and after the financial crisis.

From Table 2 note that the correlation between the levels of bank and monetary rates has decreased after 2009, while the correlation between the variations of the administered bank rates and those of the monetary rates has increased during the same period. 
Table 2. Correlation matrix between rates and their variations, in different periods.

\begin{tabular}{cccc}
\hline & $1999-2008$ & $2009-2014$ & $1999-2014$ \\
\hline$B R, M R$ & 0.95 & 0.71 & 0.96 \\
$\Delta B R, \Delta M R$ & 0.43 & 0.83 & 0.58 \\
\hline
\end{tabular}

Monetary Rates distribution
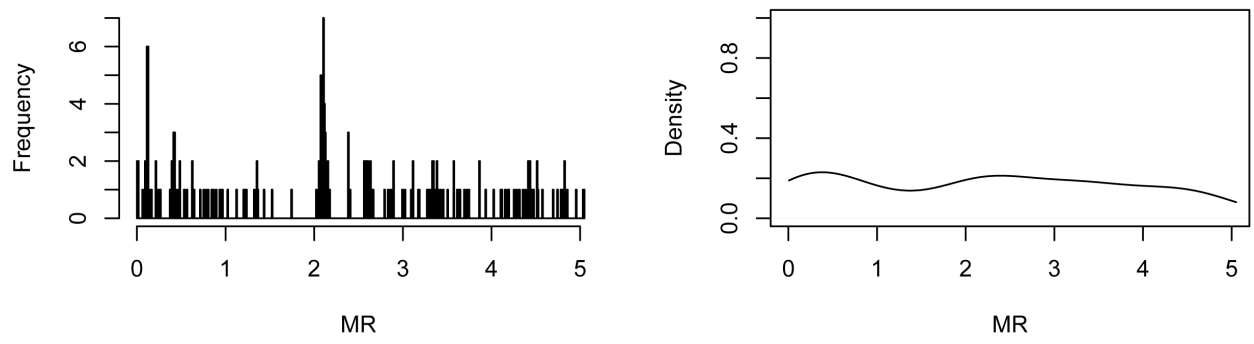

Bank Rates distribution
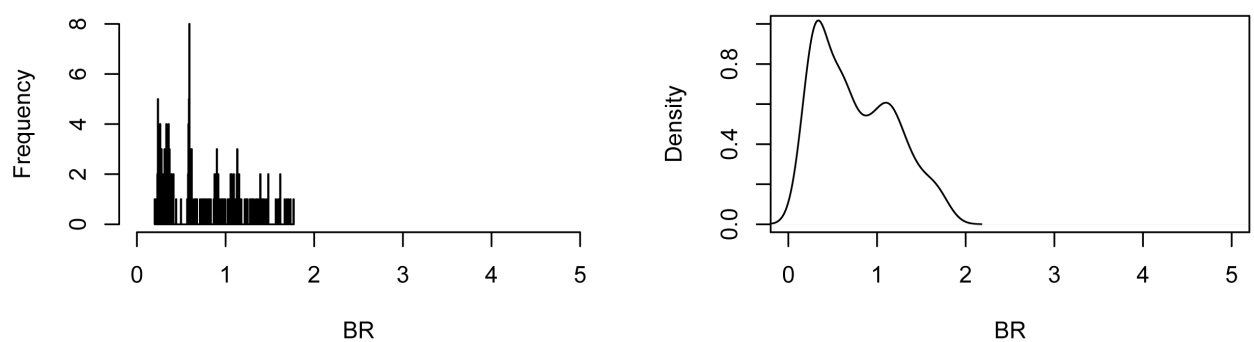

Figure 2. Distribution of the monetary and the administered bank rates.
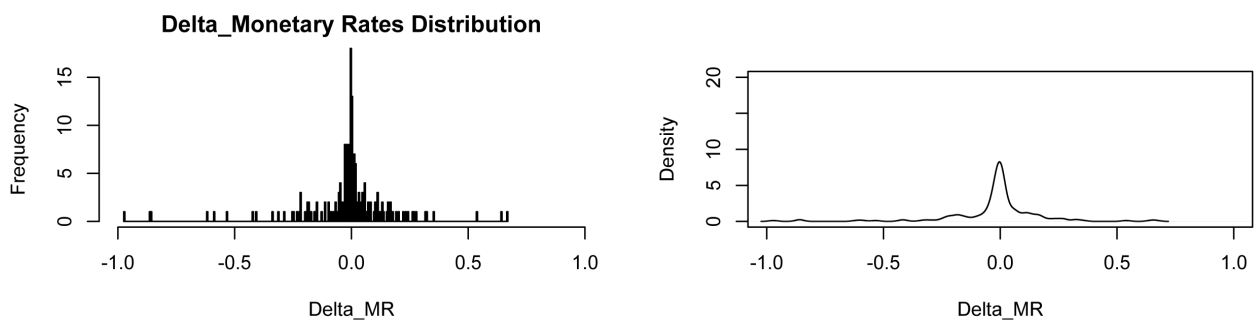

Delta_Bank Rates Distribution
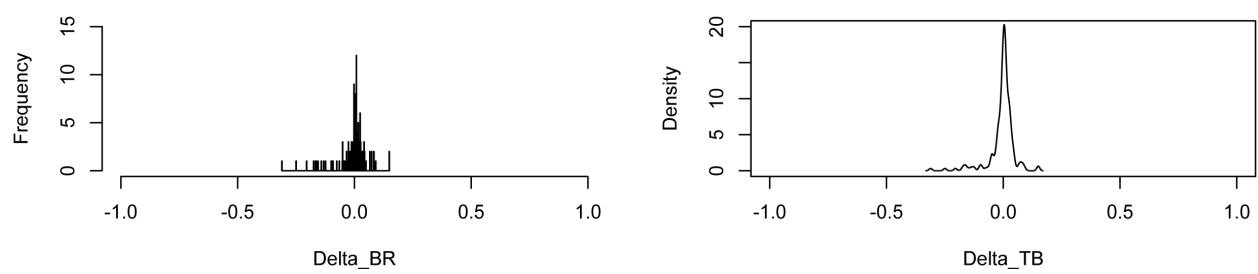

Figure 3. Distribution of the variations of monetary and administered bank rates.

\subsection{Model Estimates}

For the models proposed in Sections 2.1 and 2.2 we now show the corresponding parameter estimates, considering the following four time series: a) data from 1999 to 2007; b) data from 1999 to 2008; c) data from 2009 to 2013; d) data from 1999 to 2013. This choice of data windows is consistent with the aim of investigating the 
switching behaviour in the correlation structure of interest rates, which has occurred during the years 2008 and 2009. On the basis of this windows selection we intend to obtain predictions for the years 2008, 2009 and, finally, for the last available year, 2014. Predictions that can be compared with the actual occurred value, thus giving a measure of model predictive performance.

We now show the parameter estimates for all the considered models, including the two simple univariate linear models, and the four periods we have chosen. For each estimate we also report the corresponding t-value, and the $R^{2}$ contribution of each model.

Table 3 shows the parameter estimates for the error correction model proposed by [5] which, we recall, has two equations and, correspondingly, two $R^{2}$ measures.

From Table 3 note that, for the error correction model with two adjustment speeds, the results confirm a radical change in the relationship between the variables during the period under analysis: remembering that the long-run equation models the levels of interest rates, while the short-run equation is a function of the variations of the rates, it is clear that in the last few years the levels of the rates have become less and less important, while their variations have gained exploratory capacity.

Table 4 shows the parameter estimates for the error correction model with one adjustment speed.

From Table 4 note that the error correction model with only one adjustment speed shows results very similar to those reported in Table 3: in particular, it has similar $R^{2}$ values, meaning that this simplified version of the error correction model fits past data quite well and, therefore, it may suffice. As a further confirmation, it can be shown that the equality assumption $\delta_{1}=\delta_{2}$ in the model proposed in [5] is rejected only in one of the four considered time windows.

Table 5 shows the parameter estimates for the simple linear model in terms of the levels of the bank interest rates.

From Table 5 note that the estimates obtained with the univariate linear model for interest rates are similar to those obtained by using the long-run equation of the error correction model.

Table 3. Parameter estimates for the error correction model with two adjustment speeds.

\begin{tabular}{|c|c|c|c|c|c|c|c|c|}
\hline & \multicolumn{2}{|c|}{$1999-2007$} & \multicolumn{2}{|c|}{ 1999-2008 } & \multicolumn{2}{|c|}{ 2009-2013 } & \multicolumn{2}{|c|}{$1999-2013$} \\
\hline & Coeff. & $t$ & Coeff. & $t$ & Coeff. & $t$ & Coeff. & $t$ \\
\hline$k$ & -0.133 & -3.426 & -0.100 & -2.542 & 0.263 & 12.265 & 0.146 & 7.896 \\
\hline$\beta$ & 0.351 & 29.741 & 0.341 & 29.425 & 0.138 & 4.836 & 0.271 & 41.114 \\
\hline$\alpha$ & 0.107 & 4.412 & 0.0909 & 4.863 & 0.096 & 4.430 & 0.126 & 7.455 \\
\hline$\delta_{1}$ & -0.286 & -5.028 & -0.288 & -5.513 & -0.348 & -11.214 & -0.175 & -5.154 \\
\hline$\delta_{2}$ & -0.209 & -4.194 & -0.220 & -4.680 & -0.032 & -0.813 & -0.109 & -3.008 \\
\hline$R^{2}$ long & \multicolumn{2}{|c|}{0.893} & \multicolumn{2}{|c|}{0.880} & \multicolumn{2}{|c|}{0.287} & \multicolumn{2}{|c|}{0.905} \\
\hline$R^{2}$ short & \multicolumn{2}{|c|}{0.443} & \multicolumn{2}{|c|}{0.485} & \multicolumn{2}{|c|}{0.902} & \multicolumn{2}{|c|}{0.449} \\
\hline
\end{tabular}

Table 4. Parameter estimates for the error correction model with one adjustment speed.

\begin{tabular}{|c|c|c|c|c|c|c|c|c|c|}
\hline & & \multicolumn{2}{|c|}{ 1999-2007 } & \multicolumn{2}{|c|}{ 1999-2008 } & \multicolumn{2}{|c|}{ 2009-2013 } & \multicolumn{2}{|c|}{ 1999-2013 } \\
\hline & & Coeff. & $t$ & Coeff. & $t$ & Coeff. & $t$ & Coeff. & $t$ \\
\hline & $k$ & -0.133 & -3.426 & -0.100 & -2.542 & 0.263 & 12.265 & 0.146 & 7.896 \\
\hline & $\beta$ & 0.351 & 29.741 & 0.341 & 29.425 & 0.138 & 4.836 & 0.271 & 41.114 \\
\hline & $\alpha$ & 0.111 & 4.620 & 0.096 & 5.300 & 0.145 & 5.316 & 0.131 & 7.903 \\
\hline & $\delta$ & -0.242 & -6.388 & -0.250 & -7.132 & -0.235 & -6.707 & -0.144 & -5.721 \\
\hline$R^{2}$ & long & \multicolumn{2}{|c|}{0.893} & \multicolumn{2}{|c|}{0.880} & \multicolumn{2}{|c|}{0.287} & \multicolumn{2}{|c|}{0.905} \\
\hline$R^{2}$ & short & \multicolumn{2}{|c|}{0.437} & \multicolumn{2}{|c|}{0.480} & \multicolumn{2}{|c|}{0.822} & \multicolumn{2}{|c|}{0.444} \\
\hline
\end{tabular}


Table 5. Parameter estimates for the linear model in terms of the levels of bank interest rates.

\begin{tabular}{|c|c|c|c|c|c|c|c|c|}
\hline & \multicolumn{2}{|c|}{$1999-2007$} & \multicolumn{2}{|c|}{ 1999-2008 } & \multicolumn{2}{|c|}{$2009-2013$} & \multicolumn{2}{|c|}{ 1999-2013 } \\
\hline & Coeff. & $t$ & Coeff. & $t$ & Coeff. & $t$ & Coeff. & $t$ \\
\hline$k$ & -0.133 & -3.426 & -0.100 & -2.542 & 0.263 & 12.265 & 0.146 & 7.896 \\
\hline$\beta$ & 0.351 & 29.741 & 0.341 & 29.425 & 0.138 & 4.836 & 0.271 & 41.114 \\
\hline$R^{2}$ & \multicolumn{2}{|c|}{0.893} & \multicolumn{2}{|c|}{0.880} & \multicolumn{2}{|c|}{0.287} & \multicolumn{2}{|c|}{0.905} \\
\hline
\end{tabular}

Table 6 shows the parameter estimates for the simple linear model in terms of variations of bank interest rates.

From Table 6 it is clear that the univariate linear model for the variations of administered bank interest rates, calculated as a function of the variations of monetary rates, shows different results: first of all, the intercept term is not significant; secondly, $R^{2}$ values have an opposite trend with respect to those in Table 5, increasing during the last period. This result is a further confirmation of the changing regime after 2009.

Table 7 shows the parameter estimates for our proposed model.

Table 7 shows that our new model presents an interesting behaviour. For the whole period 1999-2013 all variables (apart from the intercept) are significant to describe the administered interest rates. But the situation changes if one concentrates on the first or on the second period: within the years 1999-2007 and 1999-2008 the variations of the monetary rates do not affect the level of bank rates; on the contrary, during the last period the only significant variable is the autoregressive component.

This is a clear evidence of the fact that, when rates are close to zero as in the last few years, administered interest rates are not affected by monetary rates, or by their variations, but, rather, they depend only on their past values.

\subsection{Predictive Performances}

After having estimated the coefficients of the different models we then predict monthly administered bank interest rates and their variations for 2008, 2009 and 2014, using a range of monetary rates scenarios, simulated from a Wiener process as previously described. In particular, for the 2014 prediction we performed the simulations by using the coefficients obtained both by considering the whole period (1999-2013) and the second part of the time range under examination (2009-2013). In Figure 4 a comparison between the predictions for 2014 (data from 1999 until 2013) obtained with the error correction model and our proposed model is shown.

As a measure of predictive performance we have calculated the root mean square errors of the predictions from all models. Here we present the prediction results in terms of variations of bank rates rather than on their levels. This because, in this case, all the predictions are more challenging, being the variations on a smaller scale.

In Table 8 the root mean square errors of the predicted variations of administered interest rates obtained with the error correction model and our proposed new model are reported.

The first column of Table 8 refers to the prediction errors for the year 2008, obtained with the two selected models, and using coefficients estimated on data from 1999 to 2007. Similarly, the second and the third column report root mean square errors for 2009 and 2014. We decided to compare predictions on these crucial years because they represent the breaking points before and after which the relationship between the rates radically changes. The objective is thus to verify whether the two models can adapt to such strong variations in the underlying economic system. Note that the last two columns both refer to estimations for 2014, but the first one uses coefficients estimated only the second period data, while the second one is based on estimations on the entire period 1999-2013.

From the analysis of Table 8 some interesting conclusions emerge: a) both models predict quite well future variations of bank interest rates; b) the error correction model works better on the whole period and, most interestingly, c) our proposed model supplies great improvements for the crucial year 2009. This means that the new model is much more flexible than the Error Correction Model, and it is able to capture essential changes in the economy not only from an estimation fit point of view, as seen in the last subsection, but also in a predictive perspective. 
Table 6. Parameter estimates for the linear model in terms of the variation of bank interest rates.

\begin{tabular}{|c|c|c|c|c|c|c|c|c|}
\hline & \multicolumn{2}{|c|}{ 1999-2007 } & \multicolumn{2}{|c|}{ 1999-2008 } & \multicolumn{2}{|c|}{ 2009-2013 } & \multicolumn{2}{|c|}{$1999-2013$} \\
\hline & Coeff. & $t$ & Coeff. & $t$ & Coeff. & $t$ & Coeff. & $t$ \\
\hline$\beta$ & 0.149 & 5.444 & 0.131 & 6.344 & 0.278 & 11.28 & 0.162 & 9.592 \\
\hline$R^{2}$ & \multicolumn{2}{|c|}{0.219} & \multicolumn{2}{|c|}{0.254} & \multicolumn{2}{|c|}{0.683} & \multicolumn{2}{|c|}{0.341} \\
\hline
\end{tabular}

Table 7. Parameter estimates for the proposed model.

\begin{tabular}{|c|c|c|c|c|c|c|c|c|}
\hline & \multicolumn{2}{|c|}{$1999-2007$} & \multicolumn{2}{|c|}{$1999-2008$} & \multicolumn{2}{|c|}{ 2009-2013 } & \multicolumn{2}{|c|}{$1999-2013$} \\
\hline & Coeff. & $t$ & Coeff. & $t$ & Coeff. & $t$ & Coeff. & $t$ \\
\hline$k$ & -0.064 & -4.394 & -0.061 & -4.561 & 0.077 & 8.498 & - & - \\
\hline$\beta$ & 0.100 & 9.369 & 0.098 & 10.992 & - & - & 0.042 & 6.205 \\
\hline$\gamma$ & - & - & - & - & - & - & 0.091 & 4.750 \\
\hline$\delta$ & 0.743 & 25.695 & 0.746 & 30.454 & 0.731 & 24.544 & 0.869 & 40.836 \\
\hline$R^{2}$ & \multicolumn{2}{|c|}{0.986} & \multicolumn{2}{|c|}{0.987} & \multicolumn{2}{|c|}{0.974} & \multicolumn{2}{|c|}{0.998} \\
\hline
\end{tabular}

Table 8. A comparison between the root mean square errors of the predictions of $\triangle B R$.

\begin{tabular}{ccccc}
\hline & 2008 & 2009 & 2014 & 2014 \\
\hline Model & $(1999-2007)$ & $(1999-2008)$ & $(2009-2013)$ & $(1999-2013)$ \\
\hline Error correction model & 0.055 & 0.171 & 0.016 & 0.003 \\
Proposed model & 0.065 & 0.069 & 0.014 & 0.018 \\
\hline
\end{tabular}
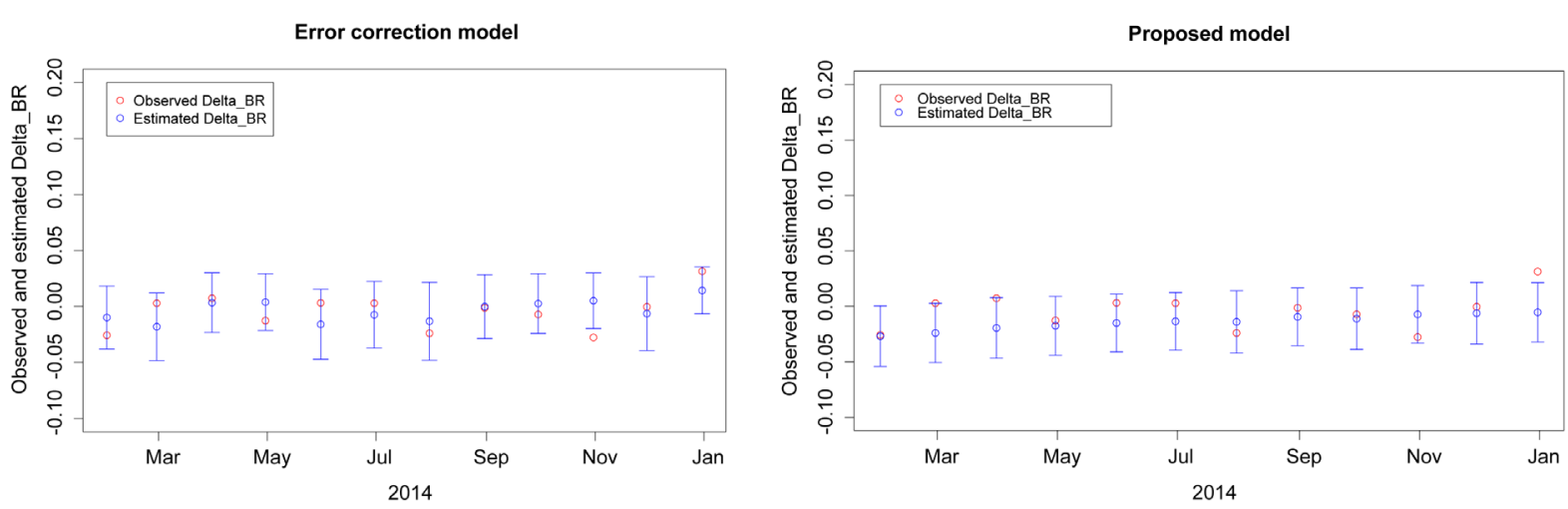

Figure 4. The estimated variations of administered interest rates for 2014, obtained with the error correction model and with our proposed model by using coefficients calculated on the whole period 1999-2013.

\section{Conclusions}

The main contribution of this paper is the understanding and improvement of the Error Correction Model, used in standard professional practice to model variations of the administered bank rates as a function of monetary rates. We add to the model a predictive methodology that allows its validation, and propose a simpler to interpret one equation model that can be seen as a special case of the ECM itself.

We have shown the implications of our proposals on data for the aggregate Italian banking sector that concerns the recent period, characterized by a substantial shift in the relationship between monetary and bank rates, with the former getting close to zero.

Future research in this topic may involve the use of time-inhomogeneous stochastic differential equations and 
dynamic linear models, in order to improve the model ability to adapt to dynamic changes.

From an applied viewpoint, it may be of interest to analyze the relationship between monetary and bank rates also on the asset side, and derive a spread measurement.

Finally, a further extension should consider the microeconomic impact of the found relationships on the probability of default of both financial and non financial corporations, enriched with a systemic correlation perspective.

\section{Acknowledgements}

We thank the Editor and the referee for their comments.

The Authors thank the Italian Association for financial risk management (AIFIRM) and the MIUR PRIN project MISURA: Multivariate statistical models for risk assessment, for financial support.

The Authors also remark that the present work actually stems from the solution of a professional risk management problem suggested by Camillo Giliberto and Igor Gianfrancesco, within the AIFIRM association.

Finally, the views expressed in this paper are those of the authors and they do not reflect the views or policies of their Institutions: Banco di Desio e della Brianza, Monte dei Paschi di Siena, University of Pavia.

\section{References}

[1] Hannan, T. and Berger, A. (1991) The Rigidity of Prices: Evidence from Banking Industry. American Economic Review, 81, 938-945.

[2] Scholnick, B. (1996) Asymmetric Adjustment of Commercial Bank Interest Rates: Evidence from Malaysia and Singapore. Journal of International Money and Finance, 15, 485-496. http://dx.doi.org/10.1016/0261-5606(96)00016-2

[3] Heffernan, S.A. (1997) Modelling British Interest Rate Adjustment: An Error Correction Approach. Economica, 64, 211-231. http://dx.doi.org/10.1111/1468-0335.00074

[4] Ballester, L., Ferrer, R., Gonzales, C. and Soto, G.M. (2009) Determinants of Interest Rate Exposure of the Spanish Banking Industry. Technical Report, University of Castilla-La Mancha.

[5] Chong, B.S., Liu, M. and Shrestha, K. (2006) Monetary Transmission via the Administered Interest Rates Channel. Journal of Banking and Finance, 30, 1467-1484. http://dx.doi.org/10.1016/j.jbankfin.2005.03.024

[6] Demirguc-Kint, A. and Huizinga, H. (1999) Determinants of Commercial Bank Interest Margins and Profitability: Some International Evidence. World Bank Economic Review, 13, 379-408. http://dx.doi.org/10.1093/wber/13.2.379

[7] Flannery, M.J. and James, C.M. (1984) The Effect of Interest Rate Changes on the Common Stock Returns of Financial Institutions. The Journal of Finance, 39, 1141-1153. http://dx.doi.org/10.1111/j.1540-6261.1984.tb03898.x

[8] Hodrick, R. and Prescott, E.C. (1997) Postwar U.S. Business Cycles: An Empirical Investigation. Journal of Money, Credit, and Banking, 29, 1-16. http://dx.doi.org/10.2307/2953682

[9] Maudos, J. and Guevara, J.F. (2004) Factors Explaining the Interest Margin in the Banking Sectors of the European Union. Journal of Banking and Finance, 28, 2259-2281. http://dx.doi.org/10.1016/j.jbankfin.2003.09.004

[10] Maudos, J. and Solis, L. (2009) The Determinants of Net Interest Income in the Mexican Banking System: An Integrated Model. Journal of Banking and Finance, 33, 1920-1931. http://dx.doi.org/10.1016/j.jbankfin.2009.04.012

[11] Gambacorta, L. (2005) How Do Banks Set Interest Rates? European Economic Review, 52, 792-819. http://dx.doi.org/10.1016/j.euroecorev.2007.06.022

[12] Gambacorta, L. and Iannotti, S. (2007) Are There Asymmetries in the Response of Bank Interest Rates to Monetary Shocks? Applied Economics, 39, 2503-2517. http://dx.doi.org/10.1080/00036840600707241

[13] Engle, R.F. and Granger, C.W.J. (1987) Co-Integration and Error Correction: Representation, Estimation, and Testing. Econometrica, 55, 251-276. http://dx.doi.org/10.2307/1913236

[14] Cocozza, R., Curcio, D. and Gianfrancesco, I. (2015) Non Maturity Deposits and Bank’s Exposure to Interest Rate Risk: Issue Arising from the Basel Regulatory Framework. Journal of Risk, 17.

[15] Basel Committee on Banking Supervision (2004) Principles for Management and Supervision of Interest Rate Risk. Technical Report, ECB. 